\title{
No association between variation in the NR4A1 gene locus and metabolic traits in white subjects at increased risk for type 2 diabetes
}

\author{
Karsten Müssig', Fausto Machicao1, Jürgen Machann2, Fritz Schick², Claus D Claussen², Norbert Stefan1, \\ Andreas Fritsche ${ }^{1,3}$, Hans-Ulrich Häring ${ }^{* 1}$ and Harald Staiger ${ }^{1}$
}

\begin{abstract}
Background: The nuclear receptor NR4A1 is implicated in metabolic regulation in insulin-sensitive tissues, such as liver, adipose tissue, and skeletal muscle. Functional loss of NR4A1 results in insulin resistance and enhanced intramuscular and hepatic lipid content. Therefore, we investigated in a cohort of white European subjects at increased risk for type 2 diabetes whether genetic variation within the NR4A1 gene locus contributes to prediabetic phenotypes, such as insulin resistance, ectopic fat distribution, or $\beta$-cell dysfunction.
\end{abstract}

Methods: We genotyped 1495 subjects (989 women, 506 men) for five single nucleotide polymorphisms (SNPs) tagging $100 \%$ of common variants (MAF $=0.05$ ) within the NR4A1 gene locus with an $r^{2}=0.8$. All subjects underwent an oral glucose tolerance test (OGTT), a subset additionally had a hyperinsulinemic-euglycemic clamp $(n=506)$. Ectopic hepatic $(n=296)$ and intramyocellular $(n=264)$ lipids were determined by magnetic resonance spectroscopy. Peak aerobic capacity, a surrogate parameter for oxidative capacity of skeletal muscle, was measured by an incremental exercise test on a motorized treadmill $(n=270)$.

Results: After appropriate adjustment and Bonferroni correction for multiple comparisons, none of the five SNPs was reliably associated with insulin sensitivity, ectopic fat distribution, peak aerobic capacity, or indices of insulin secretion (all $\mathrm{p} \geq 0.05$ ).

Conclusions: Our data suggest that common genetic variation within the NRAA1 gene locus may not play a major role in the development of prediabetic phenotypes in our white European population.

\section{Background}

In addition to peripheral insulin resistance and pancreatic beta-cell dysfunction, type 2 diabetes mellitus is also characterized by aberrant hepatic gluconeogenesis. cAMP response element-binding protein (CREB), a key regulator of hepatic gluconeogenesis, mediates its actions through transcriptional induction of the nuclear hormone receptor coactivator PGC-1 $\alpha$ (peroxisome proliferator-activated (PPAR)- $\gamma$ coactivator- $1 \alpha)$. Recently, CREBinduced activation of the NR4A orphan nuclear receptor family, including the three highly homologous isotypes, NR4A1, NR4A2, and NR4A3 (also known as Nur77,

\footnotetext{
* Correspondence: hans-ulrich.haering@med.uni-tuebingen.de

${ }^{1}$ Division of Endocrinology, Diabetology, Angiology, Nephrology, and Clinical Chemistry, Department of Internal Medicine, University Hospital of Tübingen, Otfried-Müller-Str. 10, 72076 Tübingen, Germany

Full list of author information is available at the end of the article
}

Nurr1, and Nor1), has been identified as a novel PGC-1 $\alpha$ independent mechanism for regulating hepatic gluconeogenesis [1]. The same nuclear receptors are also implicated in metabolic regulation in other insulin-sensitive tissues. NR4A1 inhibits adipocyte differentiation and regulates expression of genes linked to glucose metabolism in skeletal muscle $[2,3]$. In a very recent study, functional loss of NR4A1 was reported to result in exacerbated insulin resistance in both skeletal muscle and liver and to increase intramuscular and hepatic lipid content upon high-fat diet [4].

In light of these data, NR4A1 appears to be an attractive prediabetes candidate gene. Therefore, we studied the impact of common genetic variation within the NR4A1 gene locus on prediabetes phenotypes, including insulin resistance, ectopic fat distribution, and, as we have 
recently found an association between common polymorphisms within the NR4A3 locus and insulin release [5], also $\beta$-cell dysfunction.

\section{Methods Subjects}

The 1495 non-diabetic white subjects at increased risk of type 2 diabetes mellitus were recruited from the southern part of Germany and participated in an ongoing study on the pathophysiology of type 2 diabetes [6]. All subjects were metabolically characterized by an oral glucose tolerance test (OGTT). In randomly selected subgroups, a hyperinsulinemic-euglycemic clamp was performed, intramyocellular lipids (IMCL) and intrahepatic lipids were determined by magnetic resonance spectroscopy (MRS), peak aerobic capacity, a surrogate parameter for oxidative capacity of skeletal muscle, was measured using an incremental exercise test on a motorized treadmill (Saturn; HP-Cosmos, Traunstein, Germany) [6]. Participants gave informed written consent to the study. The protocol was approved by the local ethical committee.

\section{Genotyping}

Using the publically available phase II data of the International HapMap Project derived from a population of Utah residents with ancestry from northern and western Europe (release \#24 November 2008, http://www.hapmap.org/index.html.en), we screened in silico the complete NR4A1 gene locus spanning 15,798 bases from nucleotide 50,723,763 to nucleotide 50,739,552 (7 exons, located on human chromosome 12q13) as well as $5 \mathrm{~kb}$ of its 5 '-flanking region and $3 \mathrm{~kb}$ of its 3 '-flanking regions. Among thirteen informative single nucleotide polymorphisms (SNPs), the five SNPs rs2242107 C/T, rs1283155 $\mathrm{C} / \mathrm{T}$, rs744690 T/G, rs2603751 A/G (all located in noncoding regions of the gene locus), and rs2701124 C/T (located in the coding region resulting in a synonymous substitution) were chosen (Additional File 1), covering $100 \%$ of common variants (minor allele frequency [MAF] $=0.05$ ) within the NR4A1 gene with an $\mathrm{r}^{2}=0.8$, according to Tagger analysis http://www.broad.mit.edu/mpg/tagger. Genotyping was performed using the TaqMan assay (Applied Biosystems, Foster City, CA). The overall genotyping success rate was $99.8 \%$ (all SNPs $100 \%$, except for rs1283155: 99.1\%), and rescreening of 3\% of subjects gave $100 \%$ identical results. Genotypes were verified in a random sample of 50 subjects by bidirectional sequencing.

\section{Statistical analyses}

In order to approximate normal distribution, $\log _{e}$-transformation of the following metabolic variables was performed prior to simple and multivariate linear regression analyses: body mass index, waist circumference, fasting glucose, glucose at $120 \mathrm{~min}$. during OGTT, homeostasis model assessment of insulin resistance (HOMA-IR), OGTT- and clamp-derived insulin sensitivity index (ISI), the ratio of area under the curve (AUC) insulin to AUC glucose at $30 \mathrm{~min}$. during OGTT, the ratio of AUC Cpeptide to AUC glucose during OGTT, insulinogenic index, hepatic lipids, intramyocellular lipids (IMCL) in tibialis anterior and soleus muscles, and peak aerobic capacity. In multivariate linear regression models, the trait was chosen as dependent variable, and gender, age, body mass index (BMI), and genotype were tested as independent variables. To account for the number of SNPs analysed $(n=5)$, a Bonferroni-corrected $\alpha$-level of $p$ $<0.01$ was considered statistically significant. Bonferroni correction was not performed for the number of traits given that the traits were interrelated. The statistical software package JMP 7.0 (SAS Institue, Cary, NC) was used. Hardy-Weinberg equilibrium was tested using the $X^{2}$ test. The effect sizes detectable in the different cohorts undergoing an OGTT, a hyperinsulinemic-euglycemic clamp, and MRS were $\geq 8 \%, \geq 14 \%$, and $\geq 18 \%$ in the additive model and $\geq 20 \%, \geq 36 \%$, and $\geq 43 \%$ in the dominant model, respectively. Power calculation was performed in the additive inheritance model by F-tests and in the dominant inheritance model by two-tailed t-tests $(1-\beta>0.8)$ using G*power software available at http://www.psycho.uni-duesseldorf.de/aap/projects/gpower/.

\section{Results}

\section{Characterization and genotyping of the study population}

Characteristics of the 1495 genotyped non-diabetic subjects (989 women, 506 men) from the southwest of Germany are shown in Additional File 2. The five NR4A1 SNPs were in Hardy-Weinberg equilibrium (all p $>0.5$ ). The observed and the HapMap genotype distributions as well as linkage disequilibrium (LD) statistics are shown in Additional File 1 and Additional File 3, respectively.

\section{Associations between NR4A1 SNPs and metabolic traits}

After appropriate adjustment and Bonferroni correction for multiple comparisons, the five NR4A1 SNPs were not significantly associated with insulin sensitivity, indices of insulin secretion, ectopic fat distribution, or peak aerobic capacity (Tables 1 and 2, Additional Files 4 and 5), except for an association between rs1283155 and glucose at 120 min. of the OGTT in the additive inheritance model ( $\mathrm{p}=$ 0.0078). However, in the dominant model, this association was no longer significant $(\mathrm{p}=0.0153)$. Furthermore, no allele dose effect was seen with this association.

\section{Discussion}

Genotyping of a metabolically well-characterized population for NR4A1 SNPs revealed no reliable association of this gene locus with insulin sensitivity, insulin secretion, or ectopic fat distribution. For some traits, e.g., IMCL or 
Table 1: Associations of NR4A1 SNPs rs744690, rs2603751, and rs2242107 with metabolic parameters ( $\mathrm{n}=1495)$.

\begin{tabular}{|c|c|c|c|c|c|c|c|c|c|c|c|c|c|c|c|}
\hline SNP & & $r s 744690$ & & & & & $r s 2603751$ & & & & & rs2242107 & & & \\
\hline Genotype & TT & TG & GG & $\mathbf{P}_{\text {add }}$ & $\mathbf{P}_{\text {dom }}$ & AA & AG & GG & $\mathbf{P}_{\text {add }}$ & $\mathbf{P}_{\text {dom }}$ & CC & CT & TT & $\mathbf{P}_{\text {add }}$ & $\mathbf{P}_{\mathrm{dom}}$ \\
\hline N & 1066 & 384 & 39 & - & - & 1156 & 313 & 20 & - & - & 769 & 589 & 131 & - & - \\
\hline $\mathrm{BMI}\left(\mathrm{kg} / \mathrm{m}^{2}\right)$ & $28.4 \pm 8.1$ & $28.4 \pm 7.5$ & $28.3 \pm 7.4$ & 0.8 & 0.6 & $28.5 \pm 7.9$ & $28.8 \pm 8.2$ & $29.6 \pm 11.1$ & 0.8 & 0.5 & $28.6 \pm 7.9$ & $28.4 \pm 7.9$ & $29.2 \pm 8.8$ & 0.7 & 0.9 \\
\hline Waist circumference $(\mathrm{cm})$ & $93 \pm 17$ & $94 \pm 17$ & $92 \pm 15$ & 0.7 & 0.9 & $94 \pm 17$ & $94 \pm 18$ & $91 \pm 20$ & 0.9 & 0.9 & $94 \pm 17$ & $93 \pm 17$ & $95 \pm 18$ & 0.4 & 0.4 \\
\hline Glucose, fasting (mM) & $5.09 \pm 0.55$ & $5.10 \pm 0.55$ & $5.15 \pm 0.54$ & 0.8 & 0.5 & $5.10 \pm 0.55$ & $5.07 \pm 0.54$ & $4.96 \pm 0.53$ & 0.3 & 0.2 & $5.11 \pm 0.56$ & $5.07 \pm 0.54$ & $5.12 \pm 0.53$ & 0.7 & 0.4 \\
\hline Glucose, $120 \mathrm{~min}$. OGTT (mM) & $6.28 \pm 1.65$ & $6.11 \pm 1.67$ & $6.47 \pm 1.73$ & 0.14 & 0.08 & $6.24 \pm 1.67$ & $6.25 \pm 1.63$ & $6.09 \pm 1.46$ & 0.9 & 1.0 & $6.29 \pm 1.68$ & $6.17 \pm 1.62$ & $6.25 \pm 1.68$ & 0.4 & 0.15 \\
\hline HOMA-IR (U) & $2.44 \pm 2.11$ & $2.36 \pm 2.20$ & $2.47 \pm 2.73$ & 0.8 & 1.0 & $2.42 \pm 2.16$ & $2.40 \pm 2.12$ & $2.78 \pm 2.33$ & 0.5 & 0.4 & $2.49 \pm 2.13$ & $2.28 \pm 2.07$ & $2.65 \pm 2.57$ & 0.06 & 0.06 \\
\hline ISI, OGTT (U) & $16.7 \pm 11.1$ & $16.6 \pm 10.3$ & $15.4 \pm 7.7$ & 0.9 & 0.7 & $16.6 \pm 10.9$ & $16.6 \pm 10.7$ & $15.4 \pm 11.1$ & 0.7 & 0.7 & $16.4 \pm 11.0$ & $17.1 \pm 10.8$ & $15.7 \pm 9.8$ & 0.13 & 0.08 \\
\hline ISI, clamp (U)\# & $0.086 \pm 0.053$ & $0.086 \pm 0.061$ & $0.081 \pm 0.040$ & 0.9 & 0.6 & $0.086 \pm 0.054$ & $0.088 \pm 0.058$ & $0.046 \pm 0.013$ & 0.3 & 0.4 & $0.086 \pm 0.054$ & $0.085 \pm 0.054$ & $0.085 \pm 0.064$ & 0.7 & 0.8 \\
\hline $\begin{array}{l}\text { AUC Ins [30 min.]/AUC glc [30 min.] } \\
(p M / m M)\end{array}$ & $40.5 \pm 29.7$ & $40.8 \pm 32.0$ & $35.2 \pm 18.2$ & 0.6 & 0.5 & $40.4 \pm 29.1$ & $40.0 \pm 33.4$ & $47.5 \pm 29.4$ & 0.2 & 0.3 & $41.0 \pm 30.2$ & $38.8 \pm 27.5$ & $44.1 \pm 39.0$ & 0.8 & 0.9 \\
\hline AUC C-pep/AUC glc (pM/mM) & $317 \pm 104$ & $324 \pm 117$ & $304 \pm 82$ & 0.7 & 0.5 & $317 \pm 106$ & $324 \pm 111$ & $325 \pm 111$ & 0.6 & 0.3 & $318 \pm 105$ & $317 \pm 106$ & $331 \pm 121$ & 0.5 & 0.4 \\
\hline Insulinogenic index (pM/mM) & $50.5 \pm 40.5$ & $51.8 \pm 43.5$ & $42.2 \pm 22.8$ & 0.7 & 0.4 & $50.6 \pm 39.7$ & $49.9 \pm 45.4$ & $58.6 \pm 36.0$ & 0.4 & 0.5 & $51.2 \pm 41.4$ & $48.7 \pm 37.1$ & $55.7 \pm 52.6$ & 0.6 & 0.8 \\
\hline
\end{tabular}

Raw data are presented and given as means \pm SD. For statistical analysis, data were $\log _{-}$-transformed. AUC Insulin [30 min.]/AUC glc [30 min.], AUC C-pep/AUC glc, and insulinogenic index were adjusted for gender, age, BMl, and ISI-OGTT. Fasting glucose, glucose at $120 \mathrm{~min}$. of the OGTT, HOMA-IR, ISI-OGT, and ISI-clamp were adjusted for gender, age, and BMI. BMI and waist circumference were adjusted for gender and age. AUC - area under the curve; BMI - body mass index; C-pep - C-peptide; glc - glucose; HOMA-IR - homeostasis model assessment of insulin resistance; ISI - insulin sensitivity index; OGT - oral glucose tolerance test; $\mathrm{p}_{>}$add. - $\mathrm{p}$-value in the addidtive inheritance model; $p_{\text {dom. }}$ - $p$-value in the dominant inheritance model; SNP - single nucleotide polymorphism; $\mathrm{U}$ - units. ${ }^{\mathrm{N}} \mathrm{N}=506$. 
Table 2: Associations of NR4A1 SNPs rs2701124 and rs 1283155 with metabolic parameters $(\mathrm{n}=1495)$.

\begin{tabular}{|c|c|c|c|c|c|c|c|c|c|c|}
\hline $\begin{array}{l}N P \\
\end{array}$ & & $r s 2701124$ & & & & & $r s 1283155$ & & & \\
\hline Genotype & $\mathrm{CC}$ & CT & TT & $\mathbf{P}_{\text {add }}$ & $\mathbf{P}_{\text {dom }}$ & CC & Ст & TT & $\mathbf{P}_{\text {add }}$ & $\mathbf{P}_{\text {dom }}$ \\
\hline $\mathrm{N}$ & 1242 & 239 & 8 & - & - & 885 & 507 & 83 & - & - \\
\hline $\mathrm{BMI}\left(\mathrm{kg} / \mathrm{m}^{2}\right)$ & $28.5 \pm 7.8$ & $29.0 \pm 8.6$ & $32.3 \pm 16.1$ & 0.5 & 0.3 & $28.7 \pm 8.2$ & $28.5 \pm 7.7$ & $28.3 \pm 7.7$ & 0.8 & 0.6 \\
\hline Waist circumference $(\mathrm{cm})$ & $94 \pm 17$ & $93 \pm 19$ & $93 \pm 28$ & 1.0 & 0.8 & $94 \pm 18$ & $94 \pm 17$ & $93 \pm 16$ & 0.9 & 0.8 \\
\hline Glucose, fasting (mM) & $5.10 \pm 0.55$ & $5.06 \pm 0.54$ & $5.11 \pm 0.64$ & 0.6 & 0.3 & $5.10 \pm 0.55$ & $5.10 \pm 0.56$ & $5.04 \pm 0.48$ & 0.4 & 0.7 \\
\hline Glucose, $120 \mathrm{~min}$. OGTT (mM) & $6.25 \pm 1.66$ & $6.22 \pm 1.64$ & $5.94 \pm 1.75$ & 0.7 & 0.7 & $6.32 \pm 1.67$ & $6.07 \pm 1.62$ & $6.46 \pm 1.66$ & 0.0078 & $\begin{array}{c}0.01 \\
53\end{array}$ \\
\hline HOMA-IR (U) & $2.41 \pm 2.11$ & $2.47 \pm 2.35$ & $2.68 \pm 1.82$ & 0.5 & 0.2 & $2.51 \pm 2.30$ & $2.31 \pm 1.87$ & $2.25 \pm 2.28$ & 0.3 & 0.17 \\
\hline ISI, OGTT (U) & $16.6 \pm 10.9$ & $16.7 \pm 10.5$ & $16.1 \pm 13.2$ & 0.7 & 0.4 & $16.3 \pm 10.7$ & $16.9 \pm 10.9$ & $17.1 \pm 10.9$ & 0.2 & 0.08 \\
\hline ISI, clamp (U)\# & $0.086 \pm 0.054$ & $0.087 \pm 0.058$ & $0.054 \pm 0.001$ & 0.8 & 0.5 & $0.084 \pm 0.052$ & $0.089 \pm 0.061$ & $0.090 \pm 0.046$ & 0.7 & 0.9 \\
\hline $\begin{array}{l}\text { AUC Insulin [30 min.]/AUC glc [30 min.] } \\
(\mathrm{pM} / \mathrm{mM})\end{array}$ & $40.2 \pm 29.0$ & $41.4 \pm 35.5$ & $44.9 \pm 23.8$ & 0.9 & 0.6 & $41.3 \pm 30.8$ & $39.7 \pm 28.4$ & $37.8 \pm 34.0$ & 0.7 & 0.8 \\
\hline AUC C-pep/AUC glc (pM/mM) & $317 \pm 106$ & $329 \pm 113$ & $312 \pm 72$ & 0.2 & 0.10 & $320 \pm 103$ & $320 \pm 113$ & $304 \pm 108$ & 0.6 & 0.9 \\
\hline Insulinogenic index (pM/mM) & $50.3 \pm 39.4$ & $52.1 \pm 48.4$ & $56.1 \pm 32.8$ & 0.9 & 0.7 & $51.5 \pm 41.7$ & $50.0 \pm 39.3$ & $46.7 \pm 44.6$ & 0.8 & 0.6 \\
\hline
\end{tabular}

Raw data are presented and given as means + SD. For statistical analysis, data were log -transformed. AUC Insulin [30 min.]/AUC glc [30 min.], AUC C-pep/AUC glc, and insulinogenic index were adjusted for gender, age, BMI, and ISI-OGTT. Fasting glucose, glucose at $120 \mathrm{~min}$. of the OGT, HOMA-IR, ISI-OGTT, and ISI-clamp were adjusted for gender, age, and BMI. BMI and waist circumference were adjusted for gender and age. AUC - area under the curve; BMI - body mass index; C-pep - C-peptide; glc - glucose; HOMA-IR - homeostasis model assessment of insulin resistance; ISI - insulin sensitivity index; OGTT - oral glucose tolerance test; $p_{\text {add }}$ - $p$-value in the addidtive inheritance model; $p_{\text {dom }}$ - $p$-value in the dominant inheritance model; SNP - single nucleotide polymorphism; $U$ - units. \#ISI (clamp) data were available from 506 subjects.

Insulinogenic index was assessed as the ratio of (insulin at $30 \mathrm{~min}$. of the OGTT - fasting insulin) to glucose at $30 \mathrm{~min}$. of the OGTT. 
liver fat content, our study was sufficiently powered to detect only moderate effect sizes. Therefore, the lack of association between $N R 4 A 1$ gene variants and ectopic fat distribution has to be ultimately ruled out in larger studies with comparable measurements, such as magnetic resonance imaging (MRI) or computed tomography (CT). In line with this, recent genome-wide association studies showed that large cohorts are required to detect small effect sizes of diabetic traits, such as fasting glucose and insulin [7]. Furthermore, given that only SNPs with a MAF greater than $5 \%$ were chosen, we cannot exclude that rarer variants may be associated with prediabetic phenotypes.

\section{Conclusions}

In conclusion, our data suggest that common variation within the NR4A1 gene locus may not play a major role in the development of prediabetic phenotypes, such as insulin resistance, $\beta$-cell dysfunction, or disproportionate fat distribution, in our white European population at an increased risk for type 2 diabetes.

\section{Additional material}

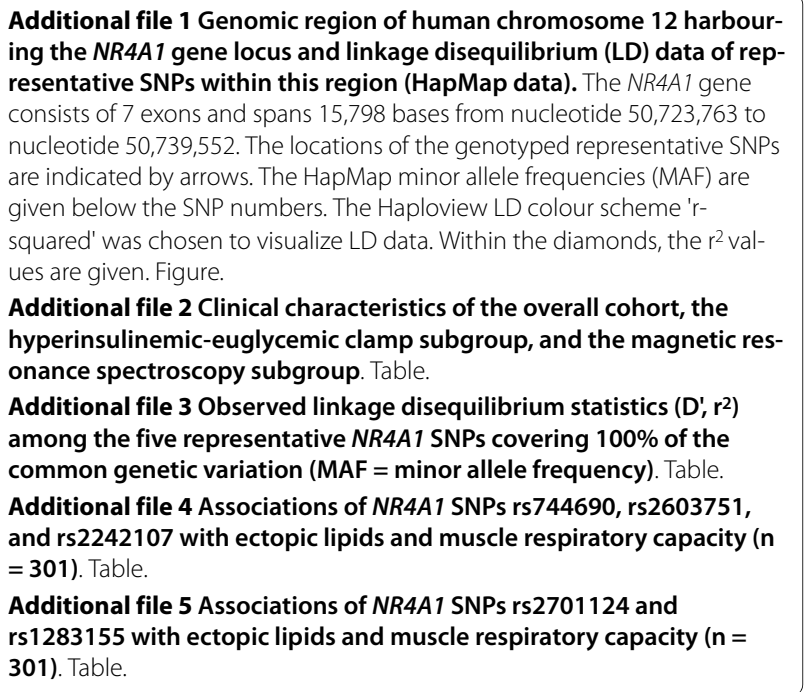

\section{Abbreviations}

BMI: body mass index; CREB: CAMP response element-binding protein; CT: computed tomography; IMCL: intramyocellular lipids; LD: linkage disequilibrium; MAF: minor allele frequency; MRI: magnetic resonance imaging; MRS: magnetic resonance spectroscopy; OGTT: oral glucose tolerance test; PGC-1a: peroxisome proliferator-activated (PPAR)- $\gamma$ coactivator-1a; SNP: single nucleotide polymorphisms.

\section{Competing interests}

The authors declare that they have no competing interests.

\section{Authors' contributions}

KM prepared all tables and figures and wrote the first draft of the manuscript. $\mathrm{KM}$ and HS were responsible for the complete statistical analysis. HS revised the manuscript. FM is responsible of the genotyping facility at the University of Tübingen. JM, FS, and CDC performed the magnetic resonance spectroscopy investigation. NS, AF and HUH acted as principal investigators for the study, and were responsible for patient and data management. All authors read and approved the final manuscript.

\section{Acknowledgements}

We thank all study participants for their cooperation. We thank the International HapMap Consortium for the public allocation of genotype data. We gratefully acknowledge the excellent technical assistance of Alke Guirguis, Melanie Weisser, Anna Bury, Heike Luz, and Roman-Georg Werner. The study was supported in part by a grant from the German Research Foundation (KFO 114/2) and a grant from the German Federal Ministry of Education and Research (BMBF) to the German Center for Diabetes Research (DZD e.V.).

\section{Author Details}

1Division of Endocrinology, Diabetology, Angiology, Nephrology, and Clinical Chemistry, Department of Internal Medicine, University Hospital of Tübingen, Otfried-Müller-Str. 10, 72076 Tübingen, Germany, 2Section on Experimental Radiology, Department of Diagnostic Radiology, University Hospital of Tübingen, Hoppe-Seyler-Str. 3, 72076 Tübingen, Germany and ${ }^{3}$ Division of Nutritional and Preventive Medicine, Department of Internal Medicine, University Hospital of Tübingen, Otfried-Müller-Str. 10, 72076 Tübingen, Germany

Received: 4 March 2010 Accepted: 4 June 2010

Published: 4 June 2010

\section{References}

1. Pei L, Waki H, Vaitheesvaran B, Wilpitz DC, Kurland IJ, Tontonoz P: NR4A orphan nuclear receptors are transcriptional regulators of hepatic glucose metabolism. Nat Med 2006, 12:1048-1055.

2. Chao LC, Zhang Z, Pei L, Saito T, Tontonoz P, Pilch PF: Nur77 coordinately regulates expression of genes linked to glucose metabolism in skeletal muscle. Mol Endocrinol 2007, 21:2152-2163.

3. Chao LC, Bensinger SJ, Villanueva CJ, Wroblewski K, Tontonoz P: Inhibition of adipocyte differentiation by Nur77, Nurr1, and Nor1. Mol Endocrinol 2008, 22:2596-2608

4. Chao LC, Wroblewski K, Zhang Z, Pei L, Vergnes L, Ilkayeva OR, Ding SY, Reue K, Watt MJ, Newgard CB, Pilch PF, Hevener AL, Tontonoz P: Insulin resistance and altered systemic glucose metabolism in mice lacking Nur77. Diabetes 2009, 58:2788-2796.

5. Weyrich $P$, Staiger H, Stancáková A, Schäfer SA, Kirchhoff K, Ullrich S, Ranta F, Gallwitz B, Stefan N, Machicao F, Kuusisto J, Laakso M, Fritsche A, Häring $\mathrm{HU}$ : Common polymorphisms within the NR4A3 locus, encoding the orphan nuclear receptor Nor-1, are associated with enhanced beta-cell function in non-diabetic subjects. BMC Med Genet 2009, 10:77.

6. Stefan N, Kantartzis K, Machann J, Schick F, Thamer C, Rittig K, Balletshofer B, Machicao F, Fritsche A, Häring HU: Identification and characterization of metabolically benign obesity in humans. Arch Intern Med 2008, 168:1609-1616.

7. Dupuis J, Langenberg C, Prokopenko I, Saxena R, Soranzo N, Jackson AU, Wheeler E, Glazer NL, Bouatia-Naji N, Gloyn AL, Lindgren CM, Mägi R, Morris AP, et al.: New genetic loci implicated in fasting glucose homeostasis and their impact on type 2 diabetes risk. Nat Genet 2010, 42:105-116.

Pre-publication history

The pre-publication history for this paper can be accessed here: http://www.biomedcentral.com/1471-2350/11/84/prepub

\section{doi: $10.1186 / 1471-2350-11-84$}

Cite this article as: Müssig et al., No association between variation in the NR4A1 gene locus and metabolic traits in white subjects at increased risk for type 2 diabetes BMC Medical Genetics 2010, 11:84 